\title{
Electrocardiogram Time Point
}

National Cancer Institute

\section{Source}

National Cancer Institute. Electrocardiogram Time Point. NCI Thesaurus. Code C83156.

The planned time point at which the electrocardiog ram assessment took place. 